\title{
La tutoría y la inclusión en la formación del profesorado de educación primaria
}

\section{Tutoring and its inclusion within primary education teacher training}

\author{
Olga María Alegre De la Rosa ${ }^{1}$ \\ oalegre@ull.edu.es \\ Remedios Guzmán Rosquete \\ rguzman@ull.edu.es \\ Carmen N. Arvelo Rosales \\ cnarvelo@ull.edu.es \\ Universidad de La Laguna, España
}

\section{Resumen:}

En la universidad del siglo XXI la acción tutorial y la diversidad del alumnado se han convertido en dos pilares que contribuyen a la calidad de la formación del profesorado. El principal objetivo de este estudio fue examinar el nivel de satisfacción que tienen los estudiantes del Grado de Educación Primaria de la Universidad de La Laguna con el rol del profesorado como tutor inclusivo en la acción docente y en la tutoría académica. Como segundo objetivo se planteó analizar si existían diferencias entre el anterior nivel de satisfacción en función del curso en el que estaban los estudiantes. Para ello se diseñó y validó una escala de satisfacción que fue administrada a una muestra de 379 estudiantes, pertenecientes a los cuatros cursos del Grado. Los resultados mostraron que estos estudiantes no tienen altos niveles de satisfacción en ninguna de las dimensiones analizadas. Se obtuvieron niveles medios de satisfacción con la tutoría

\begin{abstract}
:
Academic tutoring and diversity of students have become two pillars that contribute to the quality of teacher training in the university of the 21 st century. The main objective of this study was to examine the level of satisfaction that the students of the Degree of Primary Education at the University of La Laguna have with the role of the teacher conceived as an inclusive tutor in educational action and in academic tutoring. A second objective was to analyze whether there existed differences between previous levels of satisfaction in those stated for the year in which the students were. To this end a scale of satisfaction was designed and validated and administered to a sample of 379 students, belonging to the four undergraduate courses the degree is made of. The results showed that these students do not have high levels of satisfaction for any of the analyzed dimensions. For academic tutoring as a resource to deal with diversity, average levels
\end{abstract}

1 Dirección para correspondencia (correspondence address):

Olga María Alegre de la Rosa. Dpto. de Didáctica e Investigación Educativa. Facultad de Educación. C/ Delgado Barreto, s/n. Edificio Central. Apartado 456. 38200 San Cristóbal de La Laguna, S/C de Tenerife (España) 
académica como recurso para atender a la diversidad, siendo el nivel menor de satisfacción con el profesorado como tutor inclusivo. Las diferencias encontradas entre los cursos evidenciaron que eran los estudiantes de $4^{\circ}$ curso los que mayores niveles de satisfacción tenían con la tutoría como recurso para la diversidad y con el rol del profesorado como tutor inclusivo.

\section{Palabras clave:}

Tutoría académica; inclusión; formación del profesorado; grado de maestro en educación primaria. of satisfaction were obtained. The lowest value went to level of satisfaction with teachers seen as inclusive tutors. The differences found among the courses showed that 4th year students had higher levels of satisfaction with tutoring as a resource for diversity and with the role of teachers as inclusive tutors.

\section{Key words:}

Academic tutoring; inclusion; teacher training; degree in Primary Education.

\section{Résumé :}

Dans I'Université du XXIe siècle, I'action de tutorat et la diversité de l'ensemble des élèves sont devenus deux piliers qui contribuent à la qualité de la formation du professorat. L'objectif principal de cette étude a été d'examiner le niveau de satisfaction des étudiants en Licence d'Enseignement primaire de l'Université de La Laguna quant au le rôle du professorat comme tuteur inclusif dans l'action enseignante et dans la tutelle académique. Un second objectif a été projeté, celui d'analyser les possibles différences entre le niveau précédent de satisfaction en fonction de l'année académique des étudiants. Pour cela, une échelle de satisfaction a été élaborée et validée, pour ensuite être administrée auprès d'un échantillon de 379 étudiants, appartenant à la $4{ }^{\text {ème }}$ année de licence (Grado). Les résultats ont démontré que le niveau de satisfaction des étudiants n'est pas très élevé dans l'une des dimensions analysées. Des niveaux moyens de satisfaction ont été obtenus quant à la tutelle académique comme ressource pour répondre à la diversité. Le niveau de satisfaction quant au professorat comme tuteur inclusif, étant moindre. Les différences rencontrées entre les années académiques ont mis en évidence que les étudiants en 4 ème année de licence étaient les plus satisfaits de l'action de tutorat comme ressource pour la diversité et du rôle du professorat comme tuteur inclusif.

\section{Mots clés:}

Tutelle académique; inclusion; formation du professorat; licence de professeur dans un enseignement primaire.

Fecha de recepción: 6-3-2017

Fecha de aceptación: 27-3-2017

\section{Introducción}

El nuevo modelo de educación superior presenta la necesidad de una enseñanza centrada en el estudiante. En esa dirección surgen nuevos roles profesionales para el personal docente, un enfoque distinto de actividades y metodologías, un mayor esfuerzo por proporcionar información de los resultados de aprendizaje y verdaderos cambios en la organización de aprendizaje. En este escenario docente, el profesor universitario, 
como tutor, es un acompañante en el proceso de enseñanza-aprendizaje y un facilitador del logro de capacidades. Esta función tutorial es una actividad orientadora que realiza el tutor vinculada al proceso educativo y a la práctica docente, dentro del marco de la concepción integral de la educación y desde un enfoque inclusivo. En ese sentido, hemos considerado que el alumnado universitario que se prepara para ser maestro de Educación Primaria, debe recibir en su formación universitaria una tutoría de excelencia que le permita conocer y vivenciar metodologías diversas para desarrollar las competencias que precisa para su futuro perfil tutorial como docente. Es por ello por lo que, en este estudio, se pretende conocer la satisfacción que tienen los estudiantes del Grado de Maestro en Educación Primaria con el rol del profesorado universitario como tutor inclusivo en la acción docente y en la tutoría académica.

\section{Marco teórico}

La tutoría se ha instalado en la Enseñanza Superior como exigencia institucional y como práctica docente centrada en el aprendizaje. Supone una de las estrategias clave de adquisición de competencias, en la medida que proporciona a los estudiantes apoyo académico, personal y profesional cara a su desarrollo personal y profesional en el ámbito universitario mejorando así el proceso de enseñanza-aprendizaje. Su protagonismo reside, como señalan Martínez-Clares y cols. (2016) "tanto en el importante papel que desempeña en la formación integral de los estudiantes como en el rol que juega como uno de los ejes articuladores del nuevo enfoque educativo centrado en los alumnos y su aprendizaje" (p.81).

La acción tutorial, además de ser un elemento inherente a la función docente y a los procesos educativos (Haya y cols., 2013; Lobato y Guerra, 2016; Villar y Alegre, 2004), es una necesidad y un recurso sustancial que contribuye de manera específica a la calidad del proceso formativo (Álvarez-Pérez, 2012; Pérez-Cusó y cols., 2015) y, de forma general a la calidad y excelencia universitaria (Alegre y Villar, 2016), aportando efectos positivos para los estudiantes, el profesorado y la institución (Cano, 2009; Haya y cols., 2013).

Este cambio conceptual que durante los últimos años hemos venido presenciando sobre la tutoría universitaria, se ha evidenciado, paula- 
tinamente, con la incorporación a nivel institucional de los Planes de Acción Tutorial configurados como procesos estructurados de apoyo a la formación académica y profesional que requiere coordinación y complementariedad con otros agentes y servicios de apoyo, pero, fundamentalmente, quedar plenamente integrado en el proceso de enseñanza-aprendizaje (Martínez-Clares y cols., 2014; Lobato y Guerra, 2016) en aras a alcanzar el proyecto formativo, personal y profesional de los estudiantes (Álvarez-Pérez y cols., 2015).

Conjuntamente con este modelo de tutoría integrado en la acción orientadora convive de manera más extensiva en la institución universitaria la tutoría académica. Ambas modalidades recogidas en el Estatuto del Estudiante Universitario (Real Decreto 1791/2010, de 30 de diciembre), deja a la tutoría académica la asistencia y orientación individualizada de los estudiantes en el proceso de aprendizaje. Tradicionalmente, esta tutoría sólo se ha concebido como el tiempo que ha de cubrir el profesorado como exigencia de su dedicación profesional para atender a los estudiantes individuales o en pequeño grupo, que generalmente de forma voluntaria asisten en un horario estipulado a realizar consultas o reclamaciones vinculadas a una asignatura. No suelen utilizarse para responder a su función de orientación académica y profesional o responder a expectativas e intereses de estudiantes, o para acompañar al estudiante en el proceso de enseñanza-aprendizaje y ser facilitador del logro de competencias.

Algunos autores han obtenido resultados de interés vinculados a la importancia y visión que de las tutorías académicas tienen profesores y alumnos. Al respecto, Solagurren y Moreno (2016) recogen diversos trabajos que han investigado en los últimos años desde esta perspectiva:

Respecto al profesorado, los estudios muestran la disposición de estos a realizar labores de tutoría académica y profesional, siendo reticentes a asumir el rol de orientador personal, si bien reconocen la necesidad de dicha función (García et al., 2005). Consideran que, como tutores, han de guiar y supervisar el aprendizaje de los estudiantes pues, aunque los califican como trabajadores y responsables, también como poco autónomos, poco formados y poco curiosos, más interesados en aprobar que en aprender (Gros y Romaná, 2004). En general, el profesor no potencia un modelo tutorial determinado, desempeñando la acción tutorial que los alumnos demandan, centrada prácticamente en el componente académico y estando orientada a la superación 
de la asignatura (Lobato et al., 2005). Respecto al alumnado, un porcentaje significativo de alumnos entiende que las tutorías deben ser empleadas de forma exclusiva para aspectos académicos (Aguilera, 2010), constatándose una escasa asistencia a las mismas (Lobato et al., 2005) motivada por la ausencia de una cultura de acción tutorial (Rumbo, y Gómez, 2009). No obstante, consideran que una mayor información, orientación, motivación y comunicación con los profesores mejoraría su rendimiento académico (Tejedor y García- Valcárcel, 2007) y su desarrollo personal (Bauselas, 2003). Dejando a un lado otras facetas de la acción tutorial y centrándonos en la académica, estos estudios vienen a confirmar la inercia del modelo anterior (Michavila y García, 2003; Vieira y Vidal, 2006) en el que la resolución de dudas copa casi en exclusiva el poco uso que los alumnos hacen de las tutorías (p. 251).

Dentro del nuevo escenario en el que la docencia se centra en el estudiante, hace ya algunos años Gairín y cols. (2004) se preguntaron si podrían acabar considerándose acción docente y acción tutorial parte de un mismo esquema docente. Sobre la base de las evidencias anteriores parece que las tutorías académicas, como señalan Solaguren y Moreno (2016:251) " también han de sufrir una revalorización como un factor de calidad, que permita al profesor realizar tareas no solo de consulta y seguimiento del aprendizaje, sino también de orientación académica y apoyo de las dificultades de aprendizaje" (Tejedor y García-Valcárcel, 2007, p.251).

Deben suponer dentro del proceso formativo un nivel de intervención que contribuya a alcanzar la función que desde hace años se reclama para la tutoría universitaria, ya que también en este caso, como afirman García y cols. (2016), el profesor como tutor "Orienta al(los) estudiante(s) respecto a los problemas escolares y personales que se presentan en el trayecto de su formación, así como en la diversidad de situaciones que implica la toma de decisiones" (p.108).

Troyano y García (2009) realizaron un trabajo sobre las expectativas del alumnado, donde obtuvieron un total de 92 competencias de la figura del docente universitario como tutor, referidas a: conocimientos (14), procedimientos (37) y las actitudes personales y sociales (41). Cuando se solicitó la priorización de competencias de mayor relevancia en la función tutorial, los estudiantes consideraron que el tutor tiene que tener conocimientos de la titulación, en técnicas de estudio y conocer la realidad laboral. En cuanto a los procedimientos manifestaron que sea capaz 
de desarrollar habilidades de comunicación, de escuchar de manera activa y de orientar tanto en el itinerario académico como en el personal. Finalmente, en cuanto a las actitudes personales y sociales señalaron que el tutor tiene que ser empático, comprometido y respetuoso con el alumnado.

Tomas y cols. (2004) también habían expresado que es preciso una reorientación de la acción tutorial y para ello el docente debe conocer las características, preocupaciones, intereses, posibilidades de comprensión, es decir, el estado de desarrollo académico de sus estudiantes ya que responden a perfiles personales, sociales y académicos muy heterogéneos. En ese sentido la tutoría universitaria ha de facilitar el desarrollo de la persona durante la carrera proporcionando una atención integral y orientando los procesos de aprendizaje universitario para ayudar a la toma de decisiones durante todo su proceso formativo. En definitiva, como primer paso, es necesario que el profesor conozca las necesidades individuales de sus alumnos, les ayude en la resolución de sus problemas y valore sus posibilidades y limitaciones de manera que les pueda proporcionar la mejor orientación personalizada. Un segundo bloque de la acción docente supone saber aplicar estrategias metodológicas y de evaluación variadas en función del contenido de la materia y, sobretodo, del grupo concreto de estudiantes. Un tercer bloque propuesto es el sistema modular flexible que posibilite la autogestión del propio aprendizaje y llevar a cabo seguimientos de itinerarios a medida, con tutorías personalizadas que ayuden al avance individual de los estudiantes. Finalmente, el último bloque supone el apoyo de otros agentes para Ilevar a cabo su función tutorial, es decir, colaborar con otros profesores de distintas asignaturas del mismo curso, personal de programas concretos, etc. Ello requiere coordinación donde el centro de atención es el alumno o alumna.

Lázaro (1997), por su parte, había situado las funciones del tutor universitario en dos bloques: el intelectual y el afectivo-madurativo. Indicaba que el profesor tendría que exigir los dominios exigidos en la materia para avalar la competencia de los estudiantes y, a la vez comprendería las causas que provocan las dificultades en el aprendizaje.

En esta dirección ya se anticipaba al planteamiento que nos ocupa, la respuesta a la diversidad del alumnado, conocer y proporcionar alternativas a las dificultades en el aprendizaje para lograr el éxito de todo el alumnado en el dominio de la materia y las competencias que desa- 
rrolla. Las aulas universitarias son ámbitos de diversidad que suponen ajustes en la docencia y en la tutoría académica (Leiva y Jiménez, 2012) y donde el rol del docente como tutor implica poner en marcha todas sus capacidades profesionales para responder a las diversas necesidades de los alumnos (Amaro y cols., 2014). Como señala Alegre (2007) "atender a la diversidad del alumnado es una capacidad que los docentes universitarios han de desarrollar como indicador de su excelencia profesional y calidad docente" (p.97). Desde esta perspectiva, la tutoría también implica un proceso relacional personalizado que favorece la inclusión. El enfoque inclusivo promueve prácticas de equidad, igualdad y acceso con calidad educativa en la medida que promueve ambiente colaborativo y favorece la adquisición de capacidades en todos los estudiantes (Alegre y Villar, 2015a). La tutoría académica supone, por tanto, un modo de respuesta a la inclusión educativa y tal como Cruz (2012) manifiesta, exige conformar maneras de organización alternas tutor-tutorado, generando sistemas tutoriales que giren en torno a los intereses y necesidades de los estudiantes. Supone crear espacios inclusivos mediante aprendizaje conjunto en la dirección de incluir en las actividades tutoriales apoyos para incrementar el capital cultural de los estudiantes con experiencias significativas que formen profesionales de la educación, en nuestro caso, de la Educación Primaria no reproductivos, sino causantes de cambio e innovación.

La investigación sobre inclusión e interculturalidad con estudiantes de la Facultad de Educación de la Universidad de La Laguna de Alegre y Villar (2015b) mediante a una muestra de 1667 estudiantes a los que se administró el Cuestionario de Actitudes hacia la Inclusión y la Interculturalidad reflejó la existencia de diferencias significativas en las actitudes en función de la edad, género, experiencia en discapacidad o interculturalidad, titulación y ciclo de estudios, pero de manera genérica se reconoce la importancia de la construcción de una cultura inclusiva en el marco del aula y la tutoría, y las dificultades existentes para establecer verdaderos valores inclusivos.

Es en el binomio tutoría académica e inclusión en el que centra el presente estudio, el cual se enmarca dentro una investigación más amplia cuya finalidad ha sido analizar las competencias docentes, la inclusión y la atención a la diversidad en los estudios de Grado de Educación Primaria, en el que el rol del profesor como tutor inclusivo es fundamental. Como se desprende de lo desarrollado en esta fundamentación teórica, 
la importancia que viene recobrando la tutoría se ha visto reflejado en trabajos que han tomado como referencia la opinión de los estudiantes, aspecto contemplados en la presente investigación apoyada en los instrumentos de recogida de información utilizados por los autores de referencia.

El problema de investigación consistió en examinar el nivel de satisfacción que tienen los estudiantes del Grado de Maestro en Educación Primaria de la Universidad de La Laguna (ULL) con el rol del profesorado como tutor inclusivo en la acción docente y en la tutoría académica. Un segundo problema consistió en analizar si existían diferencias entre su nivel de satisfacción y el curso académico en el que se encontraban dichos estudiantes.

\section{Metodología de la investigación}

Para abordar las cuestiones de investigación anteriormente planteadas, se ha optado por una metodología no experimental, y se presenta como un estudio de tipo exploratorio descriptivo, mediante la aplicación de una escala de satisfacción elaborada ad hoc.

\section{Muestra}

La muestra estuvo formada por un total de 379 estudiantes (247 mujeres y 132 hombres) matriculados en los cuatro cursos del Grado de Maestro en Educación Primaria de la ULL, con un rango de edad entre 18 y 44 años $(M=21.98 ; D T=4.1)$. Un alto porcentaje de estos estudiantes (78.4\%) informaron que el Grado de Maestro en Educación Primaria había sido su primera opción de estudio. Del total de la muestra sólo el 24.5\% manifestaron que habían o estaban participado en el Programa de Acción Tutorial (POAT) propuesto por la Facultad de Educación. Respecto a la asistencia a clase y participación en las tutorías académicas, el $84.9 \%$ de estos estudiantes indicaron asistir todos los días a clase y el $30.2 \%$ informaron que asistían a menudo a las tutorías, en su mayoría (56.4\%) para resolver dudas de clase. En la tabla 1 se presentan las principales características de la muestra en función de los cuatro cursos del Grado. 
Tabla 1. Características demográficas y de participación en las tutorías en función del curso.

\begin{tabular}{|c|c|c|c|c|c|c|c|c|c|}
\hline \multicolumn{10}{|c|}{ Grado de Educación Primaria } \\
\hline & & & & & Cursos & & & & \\
\hline \multirow[t]{3}{*}{ Variable } & & \multicolumn{2}{|c|}{$1^{\circ}$} & \multicolumn{2}{|c|}{$2^{\circ}$} & \multicolumn{2}{|c|}{$3^{\circ}$} & \multicolumn{2}{|c|}{$4^{\circ}$} \\
\hline & & $N$ & $\%$ & $N$ & $\%$ & $N$ & $\%$ & $N$ & $\%$ \\
\hline & Total & 84 & 22.2 & 98 & 26 & 113 & 29.8 & 83 & 21.9 \\
\hline \multirow[t]{2}{*}{ Sexo } & Hombre & 33 & 39.3 & 39 & 39.8 & 33 & 29.4 & 27 & 32.5 \\
\hline & Mujer & 51 & 60.7 & 59 & 60.2 & 80 & 70.8 & 56 & 67.5 \\
\hline \multirow[t]{2}{*}{ Edad } & M & 19.83 & -- & 21.5 & -- & 22.7 & -- & 23.6 & -- \\
\hline & $D T$ & 3.1 & -- & 4.1 & -- & 4.6 & -- & 3.1 & -- \\
\hline \multirow{2}{*}{$\begin{array}{r}\text { Asistencia } \\
\text { a clase }\end{array}$} & $\mathrm{SI}$ & 66 & 78.6 & 81 & 82.7 & 104 & 92 & 71 & 85.5 \\
\hline & $\mathrm{NO}$ & 17 & 20.2 & 15 & 15.3 & 8 & 7.1 & 12 & 14.5 \\
\hline \multicolumn{10}{|l|}{ Tutorías } \\
\hline \multirow{2}{*}{$\begin{array}{l}\text { Particip. } \\
\text { en POAT }\end{array}$} & SI & 22 & 26.2 & 19 & 19.4 & 22 & 19.5 & 30 & 36.1 \\
\hline & $\mathrm{NO}$ & 59 & 70.2 & 79 & 80.6 & 90 & 79.6 & 53 & 63.9 \\
\hline \multirow{5}{*}{$\begin{array}{r}\text { Asistencia } \\
\text { tutoría }\end{array}$} & A menudo & 28 & 33.3 & 32 & 32.7 & 20 & 17.7 & 34 & 41 \\
\hline & Alguna vez & 15 & 17.8 & 15 & 15.3 & 1 & .9 & 15 & 18.1 \\
\hline & $\begin{array}{l}\text { Cuando son } \\
\text { obligatorias }\end{array}$ & 10 & 11.9 & 25 & 25.5 & 34 & 30.1 & 15 & 18.1 \\
\hline & $\begin{array}{r}\text { Antes de los } \\
\text { exámenes } \\
\end{array}$ & 17 & 20.2 & 18 & 18.4 & 18 & 15.9 & 12 & 14.5 \\
\hline & Nunca & 14 & 20.2 & 4 & 4.1 & 6 & 5.3 & 4 & 4.8 \\
\hline \multirow[t]{5}{*}{ Motivo } & Dudas clase & 38 & 45.2 & 62 & 63.3 & 68 & 60.2 & 43 & 51.8 \\
\hline & Dudas examen & 29 & 34.5 & 25 & 25.5 & 24 & 21.2 & 15 & 18.1 \\
\hline & T. académicos & 5 & 6 & 6 & 6.1 & 8 & 7.1 & 14 & 16.9 \\
\hline & T. personales & 5 & 6 & 4 & 4.1 & 3 & 2.7 & 9 & 10.8 \\
\hline & Otros & 7 & 8.3 & 1 & 1 & 7 & 6.2 & 2 & 2.4 \\
\hline
\end{tabular}

\section{Instrumento}

Se diseñó un cuestionario ad hoc, compuesto por un total de 29 preguntas, las cuales fueron agrupadas en dos secciones. La sección I, formada por 6 preguntas, recogía información sobre características demográficas (sexo, edad, curso), y sobre la asistencia a clase y participación en las tutorías.

La sección II la integra una escala tipo Likert, denominada Escala de Satisfacción sobre la tutoría académica y el tutor inclusivo, formada por 23 ítems con 7 alternativas de respuestas; en las alternativas de respuesta 
se ha considerado los valores de 1-3 como nivel de satisfacción bajo, el valor 4 nivel de satisfacción medio y los valores de 5-7 como niveles de satisfacción alto.

El contenido de los ítems se elaboró a partir de la bibliografía reseñada y de manera específica de la Escala de Satisfacción propuesta por Pérez-Cusó y cols. (2015) y el Cuestionario de Autoevaluación de la Función Tutorial de Villar y Alegre (2004). Asimismo, para garantizar la validez de contenido, previa a la recogida de información se solicitó la colaboración de tres académicos especialistas en la temática, los cuales analizaron el nivel de claridad, pertinencia y relevancia de cada uno de los ítems en las dimensiones en las que se incluyeron. Con la información aportada se hicieron cambios menores relacionados con la redacción de alguno de los ítems.

Con el fin de explorar cómo se agrupaban los ítems de la escala en función de las dimensiones inicialmente propuestas, se realizó un análisis de componentes principales con rotación ortogonal (Varimax). Para comprobar que los datos eran adecuados antes del factor de extracción se realizaron las pruebas de Kaiser-Meyer-Olkin (KMO) y la prueba de esfericidad de Bartlett. La media de adecuación de muestreo $(\mathrm{KMO}=$ .953) y la prueba de esfericidad de Bartlett $\left(\chi^{2}=5189.09, g l=253, p=.001\right)$ indicaron que las correlaciones entre los ítems eran adecuadas.

La matriz de componentes rotados ofreció tres factores, que conjuntamente explicaron un $61.02 \%$ de la varianza total. Para la fiabilidad del total del cuestionario se obtuvo un $\alpha$ de Cronbach de .943. El Factor I explica el $24.9 \%$ de la varianza y agrupó 10 ítems, los cuales hacen referencia al rol del profesorado como tutor inclusivo en la acción docente. El $\alpha$ de Cronbach fue de .924. El Factor II agrupó 7 ítems generales relativos a la tutoría académica, explicó el $18.9 \%$ de la varianza y el de Cronbach fue de .875. En el Factor III, se agruparon 6 ítems relacionados con la tutoría académica como recurso para atender a la diversidad, explicó el $17.2 \%$ de la varianza y el $\alpha$ de Cronbach fue de .865 . En la tabla 2 se muestra la matriz factorial después de la rotación en la que se presenta la saturación de los ítems en función de los factores. 
Tabla 2. Saturaciones factoriales de los ítems.

\begin{tabular}{cccc}
\hline & \multicolumn{3}{c}{ Factores } \\
\hline $\mathrm{N}^{\circ}$ del ítem & 1 & 2 & 3 \\
\hline 17 & .786 & .098 & .252 \\
21 & .785 & .254 & .069 \\
16 & .716 & .226 & .291 \\
18 & .702 & .118 & .206 \\
15 & .701 & .144 & .331 \\
20 & .693 & .192 & .288 \\
22 & .683 & .321 & .286 \\
19 & .635 & .282 & .386 \\
14 & .606 & .104 & .527 \\
23 & .583 & .373 & .077 \\
4 & .255 & .741 & .303 \\
6 & .234 & .709 & .080 \\
1 & .083 & .705 & .331 \\
3 & .154 & .697 & .306 \\
5 & .203 & .683 & .444 \\
2 & .304 & .635 & -.022 \\
7 & .126 & .606 & .375 \\
9 & .365 & .244 & .686 \\
12 & .305 & .260 & .655 \\
13 & .471 & .125 & .655 \\
8 & .173 & .274 & .625 \\
10 & .335 & .379 & .609 \\
11 & .227 & .430 & .571 \\
\hline & & &
\end{tabular}

\section{Procedimiento}

Para la aplicación del instrumento previamente se concertó con el profesorado de los diferentes cursos y turnos, el horario más adecuado para poder administrar el cuestionario. Posteriormente, en cada uno de los grupos se explicó el objetivo del estudio y se solicitó la colaboración voluntaria de los estudiantes, a los cuales se les entregó el cuestionario para su cumplimentación con una duración máxima de quince minutos. 


\section{Resultados}

Los datos se analizaron mediante procedimientos descriptivos e inferenciales con el SPSS-Windows V21. Se calcularon estadísticos descriptivos (medias, desviaciones típicas y porcentajes) e inferenciales para verificar la significación de las diferencias entre los tres factores y el curso (ANOVA y análisis post-hoc mediante la prueba de Scheffé). Como tamaño del efecto se informa de eta cuadrado parcial.

Con relación al primer objetivo, los análisis se realizaron en función de los tres componentes encontrados en el análisis factorial. Los resultados mostraron que los estudiantes de este Grado no tienen un alto nivel de satisfacción en ninguno de los factores encontrados. Específicamente, en los tres factores las medias totales se situaron por debajo de 4, siendo el nivel de satisfacción más alto en el Factor II relacionado con la tutoría académica $(M=3.95 ; D T=1.09)$, seguido del Factor III referido a la tutoría como recurso para atender a la diversidad $(M=3.89$; $D T=1.17)$ y, en último lugar, el Factor I en el que se agruparon la mayor parte de los ítems relacionados con el profesorado como tutor inclusivo en la acción docente $(M=3.39$; $D T=1.13)$.

\section{Nivel de satisfacción en función de los ítems agrupados en cada uno de los factores}

En cuanto al nivel de satisfacción que tienen los estudiantes sobre el rol del profesorado como tutor inclusivo (Factor I), como se refleja en la tabla 3 el análisis de los ítems reveló que la mayor parte de estos obtuvieron valores medios inferiores a 4 , lo que indicó un nivel de satisfacción bajo por parte de estos estudiantes. Así, por ejemplo, más de la mitad de los encuestados manifestaron estar nada o poco satisfechos con la coordinación entre el profesorado para atender al alumnado con necesidades educativas $(60.8 \%)$ o para mantener coordinación con otros servicios universitarios (56.2\%). Estos estudiantes también indicaron estar poco satisfechos con la motivación que da el profesorado a los estudiantes con dificultades (58\%), y con su nivel de comprensión hacia el alumnado con dificultades. que tiene el profesorado con el alumnado con dificultades $(55.7 \%)$. 
Tabla 3. Nivel de satisfacción con el profesorado como tutor inclusivo en la acción docente.

\begin{tabular}{|c|c|c|c|c|c|}
\hline \multirow[b]{2}{*}{ Factor I: ítems } & \multicolumn{5}{|c|}{ Nivel de satisfacción (\%) } \\
\hline & M & $D T$ & $\begin{array}{l}\text { Nada/ } \\
\text { poco }\end{array}$ & Medio & $\begin{array}{c}\text { Bastante/ } \\
\text { muy }\end{array}$ \\
\hline $\begin{array}{l}\text { 17. El profesorado atiende a las nece- } \\
\text { sidades educativas individuales (adap- } \\
\text { tando materiales, la evaluación, ubica- } \\
\text { ción, etc.). }\end{array}$ & 3.37 & 1.5 & 53.7 & 20.6 & 25.7 \\
\hline $\begin{array}{l}\text { 21. Existe coordinación entre el pro- } \\
\text { fesorado para dar respuesta a los es- } \\
\text { tudiantes con necesidades educativas } \\
\text { individuales. }\end{array}$ & 3.11 & 1.44 & 60.8 & 21.7 & 17.5 \\
\hline $\begin{array}{l}\text { 16. El profesorado facilita la integra- } \\
\text { ción de los estudiantes con dificultades } \\
\text { en la dinámica de clase y los trabajos } \\
\text { en grupo. }\end{array}$ & 3.51 & 1.45 & 49.3 & 23.7 & 26.9 \\
\hline $\begin{array}{l}\text { 18. En las tutorías se han tratado temas re- } \\
\text { lacionados con el desarrollo profesional. }\end{array}$ & 3.22 & 1.54 & 54.8 & 22 & 23.3 \\
\hline $\begin{array}{l}\text { 15. El profesorado mantiene contacto } \\
\text { con otros servicios de estudiantes que } \\
\text { ayudan a resolver las necesidades edu- } \\
\text { cativas individuales }\end{array}$ & 3.28 & 1.35 & 56.2 & 25.1 & 18.7 \\
\hline $\begin{array}{l}\text { 20. El profesorado motiva a todos los } \\
\text { estudiantes, en especial a los que tie- } \\
\text { nen dificultades. }\end{array}$ & 3.22 & 1.5 & 58 & 19.8 & 22.3 \\
\hline $\begin{array}{l}\text { 22. El profesorado se preocupa de bus- } \\
\text { car la mejor repuesta cuando algún } \\
\text { alumno tiene dificultades. }\end{array}$ & 3.49 & 1.43 & 50.1 & 24.8 & 25.1 \\
\hline $\begin{array}{l}\text { 19. El profesorado promueve que todos } \\
\text { los estudiantes, aunque tengan necesi- } \\
\text { dades individuales, adquieran compe- } \\
\text { tencias para su futuro profesional. }\end{array}$ & 3.7 & 1.47 & 43.3 & 28 & 28.8 \\
\hline $\begin{array}{l}\text { 14. Con el alumnado que tiene dificul- } \\
\text { tades, el profesorado se muestra com- } \\
\text { prensivo facilitándoles tiempo extra en } \\
\text { caso que lo necesiten. }\end{array}$ & 3.26 & 1.51 & 55.7 & 21.6 & 22.7 \\
\hline $\begin{array}{l}\text { 23. El profesorado proporciona infor- } \\
\text { mación sobre los recursos universita- } \\
\text { rios disponibles para los estudiantes. }\end{array}$ & 3.67 & 1.52 & 46.7 & 24.5 & 28.8 \\
\hline
\end{tabular}




\section{Nivel de satisfacción con la tutoría académica}

Como se refleja en la tabla 4, la mayor parte de los ítems agrupados en el Factor II, a excepción del ítem 2, obtuvieron puntuaciones medias en torno a 4, lo cual fue indicativo que con las tutorías académicas los estudiantes estaban medianamente satisfechos. En el análisis de los enunciados que se incluyeron en este factor, merece resaltar una mayor proporción de estudiantes que manifestaron estar medianamente o bastante satisfechos con alguno de los ítems presentados. Así, por ejemplo, cerca de la mitad del alumnado indicaron estar bastante o muy satisfechos con el trato accesible y cercano que muestra el profesorado en la tutorías, así como con el apoyo recibido por parte de este, así como, aunque en menor proporción, la disponibilidad del profesorado para atender al alumnado en las tutorías. Por el contrario, también resultó relevante que un alto porcentaje del alumnado manifestó estar nada o poco satisfechos con la organización de los horarios de las tutorías.

\section{Tabla 4. Nivel de satisfacción con la tutoría académica.}

\begin{tabular}{|c|c|c|c|c|c|}
\hline \multirow[b]{2}{*}{ Factor II: ítems } & \multirow[b]{2}{*}{ M } & \multirow[b]{2}{*}{$D T$} & \multicolumn{3}{|c|}{ Nivel de satisfacción (\%) } \\
\hline & & & $\begin{array}{l}\text { Nada/ } \\
\text { poco }\end{array}$ & Medio & $\begin{array}{c}\text { Bastante/ } \\
\text { muy }\end{array}$ \\
\hline $\begin{array}{l}\text { 4. El profesorado es competente en sus } \\
\text { funciones como tutor. }\end{array}$ & 4.01 & 1.37 & 36 & 27 & 37 \\
\hline $\begin{array}{l}\text { 6. El profesorado está disponible para } \\
\text { atenderme en el horario de tutoría. }\end{array}$ & 4.13 & 1.54 & 35.4 & 24 & 40.6 \\
\hline $\begin{array}{l}\text { 1. En general. estoy satisfecho con las } \\
\text { tutorías. }\end{array}$ & 4.13 & 1.36 & 28 & 31.1 & 38.9 \\
\hline $\begin{array}{l}\text { 3. Las tutorías me han servido para } \\
\text { mejorar mi desarrollo académico. }\end{array}$ & 3.91 & 1.43 & 39.2 & 26.2 & 34.7 \\
\hline $\begin{array}{l}\text { 5. Estoy satisfecho con el apoyo reci- } \\
\text { bido por el profesorado en las tutorías. }\end{array}$ & 4.1 & 1.45 & 32.3 & 26.5 & 41.3 \\
\hline $\begin{array}{l}\text { 2. Los horarios de tutorías están bien } \\
\text { organizados. }\end{array}$ & 3.17 & 1.46 & 61.2 & 19.3 & 19.3 \\
\hline $\begin{array}{l}\text { 7. En las tutorías el profesorado se } \\
\text { muestra accesible y cercano. }\end{array}$ & 4.27 & 1.51 & 29 & 26.9 & 44.1 \\
\hline
\end{tabular}

Con relación a los ítems agrupados en el Factor III, mediante los cuales se analizó el nivel de satisfacción sobre la tutoría académica como recurso para atender a la diversidad, los resultados mostraron una tendencia similar a la encontrada en el factor anterior; esto es el alumna- 
do informó estar medianamente satisfecho, considerando la puntuación media de los enunciados presentados. A excepción del ítems 13, en el que más de la mitad de los encuestados manifestaron estar poco satisfecho, en el resto de ítems la media se situó próxima a la puntuación 4. El mayor nivel de satisfacción se encontró en el nivel de asesoramiento que el profesorado proporciona en las tutorías sobre los objetivos a alcanzar. No obstante, aun considerando este nivel medio de satisfacción, como se puede ver en la tabla 5, en la mayor parte de los ítems los niveles de satisfacción se distribuyeron en los dos extremos de la escala, siendo el porcentaje de estudiantes que manifestaron estar nada o poco satisfechos muy similar a los que opinaron estar bastantes o muy satisfechos.

Tabla 5. Nivel de satisfacción con la tutoría como recurso para atender a la diversidad.

\begin{tabular}{|c|c|c|c|c|c|}
\hline \multirow[b]{2}{*}{ Factor II: ítems } & \multicolumn{5}{|c|}{ Nivel de satisfacción (\%) } \\
\hline & $M$ & $D T$ & $\begin{array}{l}\text { Nada/ } \\
\text { poco }\end{array}$ & Medio & $\begin{array}{c}\text { Bastante/ } \\
\text { muy }\end{array}$ \\
\hline $\begin{array}{l}\text { 9. En las tutorías el profesorado ayuda } \\
\text { a los estudiantes a planificar el trabajo, } \\
\text { prestando más atención a los que tie- } \\
\text { nen dificultades. }\end{array}$ & 3.97 & 1.49 & 37.7 & 25.6 & 36.7 \\
\hline $\begin{array}{l}\text { 12. En las tutorías el profesorado pres- } \\
\text { ta más ayuda individualizada a los es- } \\
\text { tudiantes con dificultades. }\end{array}$ & 4.1 & 1.49 & 38.3 & 23 & 38.8 \\
\hline $\begin{array}{l}\text { 13. En las tutorías el profesorado tiene } \\
\text { en cuenta las circunstancias persona- } \\
\text { les de los estudiantes con dificultades. }\end{array}$ & 3.43 & 1.56 & 52.2 & 21.4 & 26.4 \\
\hline $\begin{array}{l}\text { 8. En las tutorías puedo hablar de pro- } \\
\text { blemas personales con el profesorado. }\end{array}$ & 3.91 & 1.68 & 41.7 & 23.2 & 35.1 \\
\hline $\begin{array}{l}\text { 10. En las tutorías el profesorado pro- } \\
\text { porciona retroalimentación sobre el } \\
\text { trabajo realizado. }\end{array}$ & 4.2 & 1.42 & 39.4 & 23.6 & 37 \\
\hline $\begin{array}{l}\text { 11. En las tutorías el profesorado ase- } \\
\text { sora de manera más directa a los estu- } \\
\text { diantes con dificultades de aprendiza- } \\
\text { je sobre los objetivos a alcanzar. }\end{array}$ & 4.2 & 1.46 & 33.1 & 24.6 & 42.3 \\
\hline
\end{tabular}

\section{Nivel de satisfacción en función de los cursos.}

Para analizar si existían diferencias en el nivel de satisfacción de los estudiantes en función de los cursos, se realizó para cada uno de los 
componentes encontrados en el análisis factorial un ANOVA. Como se puede ver en la tabla 6, se encontraron diferencias significativas en el nivel de satisfacción del alumnado en el rol del profesorado como tutor inclusivo (Factor I) y en la tutoría académica como recurso para atender a la diversidad (Factor III). Por el contrario no se encontraron diferencias significativas en el nivel de satisfacción con la tutoría académica (Factor II).

Tabla 6. Medias, desviaciones típicas y significaciones estadísticas en los tres factores.

\begin{tabular}{lccccccc}
\hline & \multicolumn{5}{c}{ CURSOS } & & \\
\cline { 2 - 5 } & $1^{\text {o }}$ & $2^{\text {o }}$ & $3^{\text {o }}$ & $4^{\circ}$ & $F(3.375)$ & $p$ & $\eta^{2}$ \\
\cline { 2 - 5 } & $M(D T)$ & $M(D T)$ & $M(D T)$ & $M(D T)$ & & & \\
\hline $\begin{array}{l}\text { Tutor } \\
\text { inclusivo }\end{array}$ & $34.8(11.7)$ & $31.4(12.1)$ & $32.4(9.1)$ & $37.8(11.8)$ & 5.88 & $<.001 .045$ \\
$\begin{array}{l}\text { Tutoría } \\
\text { académica }\end{array}$ & $27.3(7.8)$ & $26.3(8.2)$ & $28(7.1)$ & $29.3(7.2)$ & 2.41 & .066 & .019 \\
$\begin{array}{l}\text { Tutoría } \\
\text { diversidad }\end{array}$ & $22.1(6.7)$ & $21.9(7.7)$ & $23.6(6.4)$ & $26(6.5)$ & 6.73 & $<.001 .051$ \\
\hline
\end{tabular}

Los análisis a posteriori utilizando la prueba Scheffé (ver tabla 7), indicaron que las diferencias en el nivel de satisfacción con el profesorado como tutor inclusivo se producían entre los estudiantes de $2^{\circ}$ y $4^{\circ}$ curso, así como entre los estudiantes de $3^{\circ}$ y $4^{\circ}$ curso; fueron los estudiantes de $4^{\circ}$ curso los que manifestaron tener un mayor nivel de satisfacción. Respecto a las diferencias encontradas en la tutoría académica como recurso para atender a la diversidad, los análisis post-hoc indicaron que las diferencias se producían entre los estudiantes de $1^{\circ}$ y $4^{\circ}$ curso, y entre los de $2^{\circ}$ y $4^{\circ}$ curso, siendo igualmente en ambos casos el nivel de satisfacción superior por parte de los estudiantes del último curso 
Tabla 7. Diferencias post-hoc entre cursos.

\begin{tabular}{ccccc}
\hline Factor & Pares & Diferencia medias & Error típico & $\boldsymbol{p}$ \\
\hline \multirow{4}{*}{ Tutor inclusivo } & $1^{\circ}-2^{\circ}$ & .339 & .165 & .242 \\
& $1^{\circ}-3^{\circ}$ & .240 & .160 & .527 \\
& $1^{\circ}-4^{\circ}$ & -.299 & .172 & .306 \\
& $2^{\circ}-3^{\circ}$ & -.099 & .153 & .936 \\
& $2^{\circ}-4^{\circ}$ & -.639 & .166 & .002 \\
& $3^{\circ}-4^{\circ}$ & -.539 & .161 & .011 \\
& & & & \\
Tutoría atención & $1^{\circ}-2^{\circ}$ & .019 & .170 & 1.00 \\
a la diversidad & $1^{\circ}-3^{\circ}$ & -.269 & .165 & .451 \\
& $1^{\circ}-4^{\circ}$ & -668 & .178 & .003 \\
& $2^{\circ}-3^{\circ}$ & -.289 & .158 & .344 \\
& $2^{\circ}-4^{\circ}$ & -.688 & .171 & .001 \\
& $3^{\circ}-4^{\circ}$ & -.399 & .166 & .125 \\
\hline
\end{tabular}

\section{Discusión y conclusiones}

Se obtuvo que los estudiantes están medianamente satisfechos con la tutoría académica, produciéndose los menores niveles de satisfacción con el profesorado como tutor inclusivo. El análisis de los ítems que se agruparon en cada uno de los factores confirmó esta tendencia general.

Si bien el nivel de satisfacción con la tutoría académica denotó niveles más altos de satisfacción en comparación con el resto de factores analizados, ello no implica afirmar que los estudiantes del estudio tengan una satisfacción adecuada o que estén altamente satisfechos con las tutorías, ya que como se desprendió de los resultados los valores se situaron en torno al punto medio. Además, el que no se hayan encontrado diferencias significativas entre los estudiantes de los diferentes cursos en la tutoría académica nos lleva a interpretar que estos resultados son consistentes y permanecen estables con independencia de la experiencia o años en la universidad.

Del análisis de los enunciados incluidos en esta dimensión o factor, se desprende que los estudiantes están más satisfechos con el perfil del profesorado universitario como tutor que con otros elementos relativos a la organización de la tutoría, como por ejemplo los horarios de las mismas. Estos resultados van en la dirección de lo encontrado por Pérez- 
Cusó, y cols. (2015) en el que analizando la satisfacción de estudiantes universitarios con la tutoría, también encontraron una mayor satisfacción con el tutor que con la organización y contenidos de la tutoría. Ello confirma que variables personales como la cercanía, el trato personal, la empatía que algunos autores han asociado al perfil que deben tener los tutores (Cruz, 2012; Gairín y cols., 2004; Troyano y García, 2009) contribuyen a incrementar los niveles de satisfacción de los estudiantes. Por el contrario, era esperable que en las cuestiones organizativas, principalmente cuando hacen referencia a horarios previamente establecidos en las que el alumnado generalmente no participa, no se encontraran buenos niveles de satisfacción, ya que como se ha encontrado en otros estudios esta es una de las debilidades más comunes manifestadas por los estudiantes (Martínez-Clares y cols., 2016; Rubio y Martínez, 2012).

Esta tendencia a estar medianamente satisfechos con la tutoría académica, se confirmó igualmente cuando esta se utiliza como recurso para atender a la diversidad del alumnado. A pesar de que la naturaleza más individualizada de esta modalidad tutorial posibilita más actuaciones dirigidas a guiar y orientar, desde una perspectiva integral, a los estudiantes con dificultades de aprendizaje a lo largo de periodo formativo, tampoco se encontró en esta dimensión un alto nivel de satisfacción. Los estudiantes manifestaron estar más satisfechos con el profesorado en la tutorización de los aspectos académicos que en los elementos de tipo personal. Por ejemplo, un porcentaje considerable del alumnado manifestó estar bastante o muy satisfechos con el asesoramiento más directo y el trato individualizado que el profesorado proporciona a los estudiantes con dificultades de aprendizaje. Sin embargo, este grado de satisfacción se produce en actuaciones dirigidas a dar una mayor retroalimentación sobre el trabajo realizado o sobre los objetivos a alcanzar. Contrariamente, indicaron estar poco satisfechos con el profesorado a la hora de tener en cuenta circunstancias personales o hablar de sus problemas personales. Como señalaron Tomás y cols. (2004) para poder proporcionar una orientación desde la dimensión personalizada es necesario que el profesor conozca las necesidades individuales de sus alumnados. En este sentido nuestros resultados confirmaron lo que otros autores han encontrado respecto a que aunque el profesorado reconoce la necesidad de asumir el rol de orientador personal es algo para lo que más reticente se muestra (García y cols., 2005, citados por Solagurren y Moreno (2016). 
Los resultados encontrados sobre el rol de profesorado como tutor reflejan una escasa cultura de la acción tutorial desde la perspectiva analizada en el presente estudio. Al respecto merece señalar que uno de los elementos que menor satisfacción produjo fue la coordinación entre el profesorado y con otros servicios de apoyo para atender al alumnado con necesidades educativas. Como se desprende del estudio realizado por Amaro y cols. (2014) la coordinación con otros docentes favorece que el rol del profesorado como tutor inclusivo se vaya consolidando en la universidad. Asimismo, la ausencia de coordinación con otros servicios de apoyo para estos estudiantes puede implicar la no consideración en el proceso de enseñanza-aprendizaje de todos los elementos y recursos que requiere la adecuada respuesta de los estudiantes con necesidades educativas individuales.

Por tanto, si bien los niveles de satisfacción encontrados confirman las inercias que siguen estando presentes en la tutoría académica universitaria, esperamos que en el contexto institucional en el que se desarrolló este estudio, los resultados encontrados sirvan para que en la formación de los futuros maestros de educación primaria se desarrollen competencias que asocien la tutoría y la diversidad al proceso integral de enseñanza-aprendizaje y más, habida cuenta que el perfil que se exige para el tutor en Educación Primaria tiene que ver con la capacidad para establecer relaciones e interacciones personales con los alumnos y las familias de éstos, la capacidad de saber negociar y mediar en los conflictos que se plantean en el vida escolar y conocer en profundidad el planteamiento curricular del nivel educativo de sus alumnos, para ser capaz de integrar los diferentes elementos del currículo y personalizarlos en cada uno de sus alumnos.

Se es consciente de que es difícil romper con la cultura que sobre la tutoría académica sigue imperando en la universidad, y que el cambio no suele generalizarse con voluntarismos, sino con apuestas decididas y comprometidas a niveles institucionales. Sin embargo, propuestas innovadoras desde la práctica pueden contagiar el sentido integrador de la tutoría universitaria. Para ello es necesario fusionar los dos niveles de intervención tutorial que actualmente coexisten y partir de necesidades sentidas, percibidas y consensuadas con el otro protagonista de la acción tutorial, que a nivel personal, académico y profesional tienen los estudiantes desde su diversidad. 


\section{Referencias bibliográficas}

Alegre, O.M. y Villar, L.M. (2016). Etnografía y narrativa de un profesor universitario en e-Portafolios. Revista Internacional de Formaçao de Professores (RIFPF), 1(1), 50-66.

Alegre, O.M. y Villar, L.M. (2015a). Inclusión e interculturalidad. Un estudio en el marco de la enseñanza universitaria. Revista nacional e internacional de educación inclusiva, 8(3), 12-29.

Alegre, O.M. y Villar, L.M. (2015b). (Eds.) Research on University Teaching and Faculty Development. New York: Nova Publishers.

Alegre, O.M. (2007). Atienda a la diversidad del alumnado. En L.M. Villar (Coord.), Programa para la mejora de la docencia universitaria (pp.97-125). Madrid: Pearson/ Prentice-Hall.

Álvarez-Pérez, P. (2012). Los planes de tutoría de carrera: una estrategia para la orientación al estudiante en el marco del EEES. Educar, 48(2), 247-266.

Álvarez-Pérez, P., López, D. y Pérez, D. (2015). El Alumnado Universitario y la Planificación de su Proyecto Formativo y Profesional. Actualidades Investigativas en Educación, 15(1),1-24.

Amaro, C., Méndez, J. y Mendoza, F. (2014). Un estudio de las características profesionales del docente universitario para atender a la diversidad. Revista Latinoamericana de Educación Inclusiva, 8(2),199-216.

Cano, R. (2009). Tutoría universitaria y aprendizaje por competencias ¿Cómo lograrlo? Revista Electrónica Interuniversitaria de Formación del Profesorado, 12(1), 181-204.

Cruz, G. (2012). Inclusión en la Educación Superior: de la atención a la diversidad al facultamiento del estudiantado. Revista científica electrónica de Educación y Comunicación en la Sociedad del Conocimiento, 12, 216-230.

Gairin, J., Feixas, M., Guillamón, C. y Quinquer, D. (2004). La tutoría académica en el escenario europeo de la Educación Superior. Revista Interuniversitaria de Formación del Profesorado, 18(1), 61-77.

García, B., Ponce, S., García, H., Caso, J., Morales, C., Martínez, Soto, Y., Serna, A., Islas, D. Martínez, S. y Aceves, Y. (2016). Las competencias del tutor universitario: una aproximación a su definición desde la perspectiva teórica y de la experiencia de sus actores. Perfiles Educativos, 38(151),104-122.

Haya, I., Calvo, A. y Rodríguez-Hoyos, C. (2013). La dimensión personal de la tutoría universitaria. Una investigación cualitativa en la Universidad de Cantabria, España. Revista Española de Orientación y Psicopedagogía, 24(3), 98-113.

Lázaro, A.J. (1997). La función tutorial en la formación docente. Revista Interuniversitaria de Formación del Profesorado, 28, 93-108.

Leiva, J., y Jiménez, A. (2012). La educación inclusiva en la universidad del siglo XXI: un proceso permanente de cambio. Revista Electrónica de Investigación y Docencia, 8, 41-62.

Lobato, C. y Guerra, N. (2016). La tutoría en la educación superior en Iberoamérica: Avances y desafíos. Educar, 57(2), 379-398.

Martínez-Clares, P., Martínez-Juárez, M., y Pérez-Cusó, J. (2016). ¿Cómo avanzar en la 
La tutoría y la inclusión en la formación del profesorado de educación primaria Olga María Alegre De la Rosa, Remedios Guzmán Rosquete y Carmen N. Arvelo Rosales

tutoría universitaria? Estrategias de acción: los estudiantes tienen la palabra. Revista Española de Orientación y Psicopedagógica, 27 (2), 80-98.

Martínez-Clares, P., Pérez-Cusó, J. y Martínez-Juárez, M. (2014). Una (re)visión de la tutoría universitaria en los estudios de Grado. REDU: Revista de Docencia Universitaria, 12(1), 269-305.

Pérez-Cusó, J., Martínez-Clares, P., y Martínez-Juárez, M.(2015). Satisfacción del estudiante universitario con la tutoría. Diseño y validación de un instrumento de medida. Estudios sobre Educación, 29, 81-101.

Real Decreto 1791/2010, de 30 de diciembre, por el que se aprueba el Estatuto del Estudiante Universitario. (BOE de 31 de diciembre de 2010).

Solaguren, M. y Moreno L. (2016). Escala de actitudes de los estudiantes universitarios hacia las tutorías académicas. Educación XX1, 19(1), 247-266.

Tejedor, F.J. y García-Valcárcel, A. (2017). Causas del bajo rendimiento del estudiante universitario (en opinión de los profesores y alumnos). Propuestas de mejora en el marco del EEES. Revista de Educación, 342, 443-473.

Tomás, M., Armengol, C., Borrel, N., Castro, D., Gairín, J. y Feixas, M. (2004). La acción tutorial docente en el contexto de la Universidad Europea: Percepción del profesorado encuestado por el grupo CCUC. IV Seminario de Expertos CCUC. Universidad Autónoma de Barcelona.

Troyano, Y. y García, A.J. (2009). Expectativas del alumnado sobre el profesorado tutor en el Espacio Europeo de Educación Superior. REDU. Revista de Docencia Universitaria, 3, 1-8.

Villar, L.M. y Alegre, O.M. (2004). Manual para la Excelencia en la Enseñanza Superior. Madrid: McGraw-Hill. 
